\title{
Viscoelastic behaviour of macro-defect-free cement
}

\author{
TUN-JEN CHU, R. E. ROBERTSON* \\ Macromolecular Science and Engineering Center, and Department of Materials Science and \\ Engineering, The University of Michigan, Ann Arbor, MI 48109, USA
}

The viscoelastic behaviour of macro-defect-free (MDF) cement was studied by dynamic mechanical analysis. MDF specimens with various moisture contents in the range $1.70 \%-3.20 \%$ moisture, were measured at $1 \mathrm{~Hz}$ as a function of temperature from $34-96^{\circ} \mathrm{C}$ and as a function of frequency from $0.05-5 \mathrm{~Hz}$ at $34^{\circ} \mathrm{C}$. The viscoelasticity of MDF was found to be affected by moisture content through its plasticizing effect on the PVA binder. Time-moisture and temperature-moisture superposition of the shear moduli were found to be possible for MDF, and linear relationships between log time and linear moisture and between temperature and moisture were found. How the microstructure of MDF affects the viscoelastic response is also discussed through mechanical models. A comparison of the models with known experimental data suggests that the viscoelastic response arises from both direct connections between the inorganic particles and from connections through the polymer binder. Inorganic links are estimated to connect $45 \%$ of the inorganic phase.

\section{Introduction}

Macro-defect-free (MDF) cement is a high-strength, high-modulus, high-toughness composite made from calcium aluminates (CA) and partially hydrolysed poly(vinyl acetate) (PVAc) by a process in which a high shear field is used [1]. MDF cement is also moisture-sensitive. The modulus has been reported to decrease by approximately $30 \%$ [2] and the strength has been reported to decrease by $50 \%$ [3] when MDF cement is exposed to moisture. In addition, the mechanical properties of MDF are time-dependent, and the time dependency has been found to be magnified by moisture $[4,5]$. The polymer phase is implicated in both the moisture sensitivity and the time dependency. The poly(vinyl acetate) is probably completely hydrolysed to poly(vinyl alcohol) (PVA) after processing, and PVA is known to swell and dissolve in water [6]. Though implicated in the moisture sensitivity and viscoelastic behaviour of MDF cement, the actual role of the PVA has not been defined. To do this, we have measured the effect of moisture on the viscoelastic properties of MDF cement over a range of frequencies, temperatures, and moisture contents and have compared this with the behaviour of PVA under similar conditions and with the known structure of the cement.

The structure of MDF cement has been under active study since its development a decade ago [7-11]. It is generally believed that the cement particles (calcium aluminates) are bonded together by PVA that has been altered or cross-linked by ions released from the cement $[7,8]$. In addition, the unhydrated cement particles are surrounded by a thin "interphase" layer distinct from the rest of the PVA that is composed of small hydrate crystals embedded in the PVA [11].

\section{Experimental procedure}

The preparation of MDF cement followed a standard optimized recipe containing the following materials: $150 \mathrm{~g}$ high-alumina cement (Secar 71, Lafarge), $10.5 \mathrm{~g}$ PVA (Gohsenol KH-17s, Nippon Gohsei), 1.05 g glycerin as a plasticizer, and $16 \mathrm{ml}$ distilled water. The mixture was processed using a twin-roll mill with multiple shearing and calendaring procedures. The final flexible sheet was warm pressed at $80^{\circ} \mathrm{C}$ for $10 \mathrm{~min}$ at $5 \mathrm{MPa}$. The sheet was stiffened by the warm pressing and was further cured between two aluminium plates in a forced-air oven at $80^{\circ} \mathrm{C}$ for $24 \mathrm{~h}$.

Rectangular specimens with the dimensions of $70 \mathrm{~mm} \times 12 \mathrm{~mm} \times 2.3 \mathrm{~mm}$ were cut from a MDF plate with a slow-speed diamond saw. The thickness, width, and gauge length of each specimen were measured with a micrometre prior to analysis. The moisture contents of the MDF specimens were controlled by timed exposures to moisture-saturated air (about $98 \%$ r.h.) at $24^{\circ} \mathrm{C}$ or a vacuum. Specimen weights were recorded just before and after the analysis. Moisture contents were obtained from the weight loss after drying in a vacuum oven at $110^{\circ} \mathrm{C}$ until the weight stabilized (5-7 days).

A dynamic mechanical analyser (DMA, DuPont model 983) was used to measure the dynamic response of MDF over a range of moisture contents. Specimens

* Author to whom all correspondence should be addressed. 
were mounted on a pair of pivot arms and a torque of $0.9 \mathrm{Nm}$ was applied to the clamp screws. Flexure was applied through the oscillation of one of the arms. Two tests were conducted on each specimen: first was an analysis at a series of fixed frequencies, $0.05,0.1,0.5,1,5$ and $10 \mathrm{~Hz}$, at $34^{\circ} \mathrm{C}$; the second was a temperature-scan programme with a window from $34-96^{\circ} \mathrm{C}$ at a heating rate of $2{ }^{\circ} \mathrm{Cmin}^{-1}$ at $1 \mathrm{~Hz}$. The window was kept below $100^{\circ} \mathrm{C}$ to avoid evaporating the moisture in the specimen. The oscillation amplitude was $0.2 \mathrm{~mm}$ for all tests. A length correction at resonance for each specimen was run prior to the analysis at room temperature. Both bending and shear stresses were induced in this flexural mode oscillation. Shear storage modulus, $G^{\prime}$, shear loss modulus, $G^{\prime \prime}$, and loss tangent, $\tan \delta=G^{\prime \prime} / G^{\prime}$ vus sus frequency or temperature were plotted.

\section{Results}

The shear storage moduli, $G^{\prime}$, of MDF cement specimens measured by the dynamic mechanical analyser at $34^{\circ} \mathrm{C}$ as a function of oscillation frequency for various moisture contents, are shown in Fig. 1. The storage modulus decreased as the frequency decreased and as the moisture content increased. $G^{\prime}$ decreased with decreasing frequency from $17.3 \mathrm{GPa}$ to $16.7 \mathrm{GPa}$ for the specimen with the lowest moisture content $(1.70 \%)$ and from $15.3 \mathrm{GPa}$ to $14.5 \mathrm{GPa}$ for the highest moisture content $(3.20 \%)$. These shear moduli are consistent with previous measurements of approximately $17 \mathrm{GPa}$ for dry MDF [7] and $12 \mathrm{GPa}$ for wet MDF [2].

The loss tangents, $\tan \delta$, for MDF specimens measured by the dynamic mechanical analyser at $34^{\circ} \mathrm{C}$ as a function of oscillation frequency for the same moisture contents as above, are shown in Fig. 2. As the frequency decreased, $\tan \delta$ increased, from 0.010 to 0.012 for the moisture content of $2.39 \%$ and from 0.016 to 0.023 for the highest moisture content $(3.20 \%)$, for example. The measured increase in $\tan \delta$ was not found to be monotonic in moisture content.

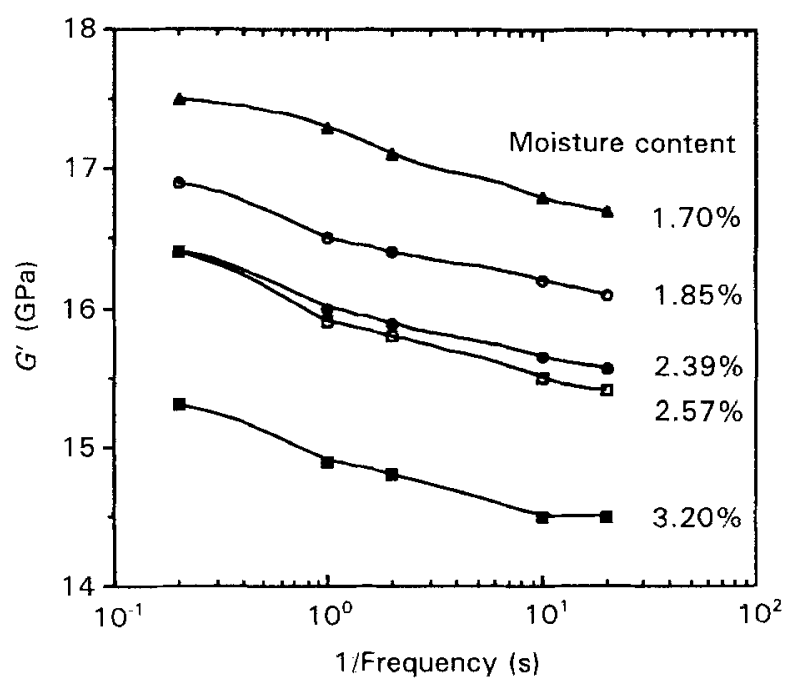

Figure 1 Shear storage moduli, $G^{\prime}$, of MDF cement versus frequency at $34^{\circ} \mathrm{C}$ for various moisture contents.

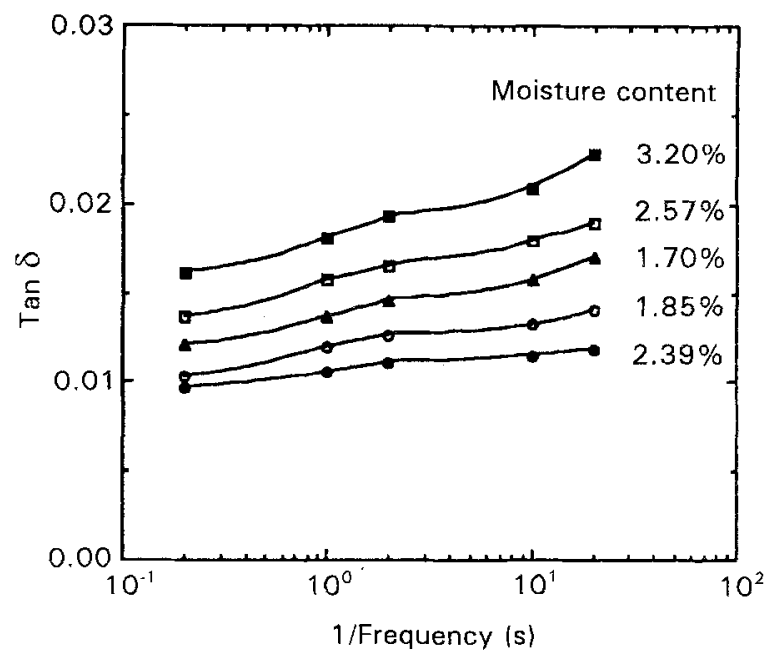

Figure 2 Loss tangents, $\tan \delta$, of MDF cement versus frequency at $34^{\circ} \mathrm{C}$ for various moisture contents.

This may have been caused by differences in clamping of these rigid specimens.

When measured against temperature (Figs 3 and 4), the storage modulus was found to decrease and the loss tangent to increase as temperature increased. Again, the responses depended on moisture content. For these temperature-scan experiments, the moisture content changed during the scan, with most of the change occurring above $60^{\circ} \mathrm{C}$. Although the specimens with the least moisture content were minimally affected, the moisture loss for the three highest moisture content specimens was about $10 \%, 12 \%$, and $14 \%$, respectively. The moisture contents at the beginning and end of the temperature scans are indicated in Figs 3 and 4.

$G^{\prime}$ decreased from $17.3 \mathrm{GPa}$ to $13.3 \mathrm{GPa}$ for the lowest moisture content specimen and from $15 \mathrm{GPa}$ to $8.5 \mathrm{GPa}$ for the highest moisture content. Tan $\delta$ increased from 0.010 to 0.030 with "normal" MDF cement (having $2.39 \%$ moisture at the beginning of the experiment) and from 0.016 to 0.045 for the highest moisture content, for example. The loss tangent increased monotonically in the temperature span of

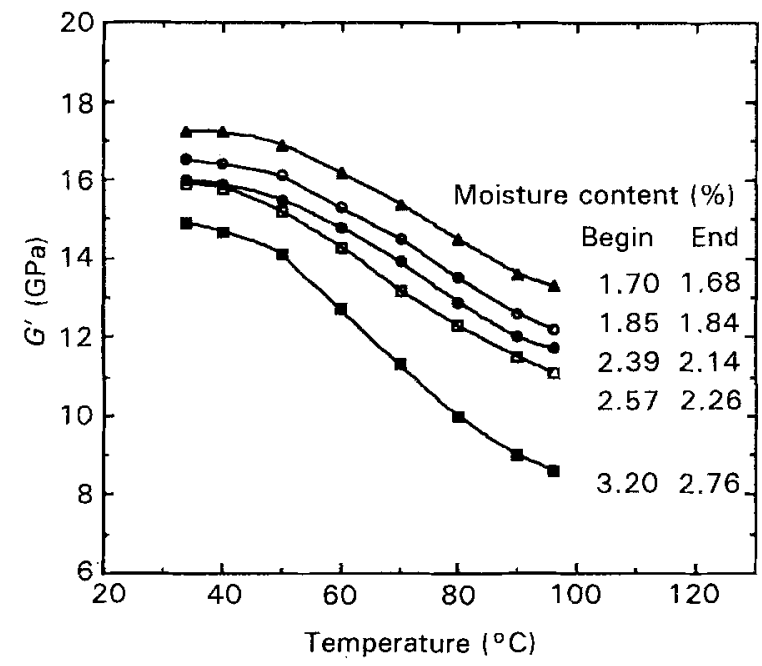

Figure 3 Shear storage moduli, $G^{\prime}$, of MDF cement versus temperature at an oscillation frequency of $1 \mathrm{~Hz}$ for various moisture contents. (The moisture contents at the beginning and end of the scan from low to high temperature are indicated.) 


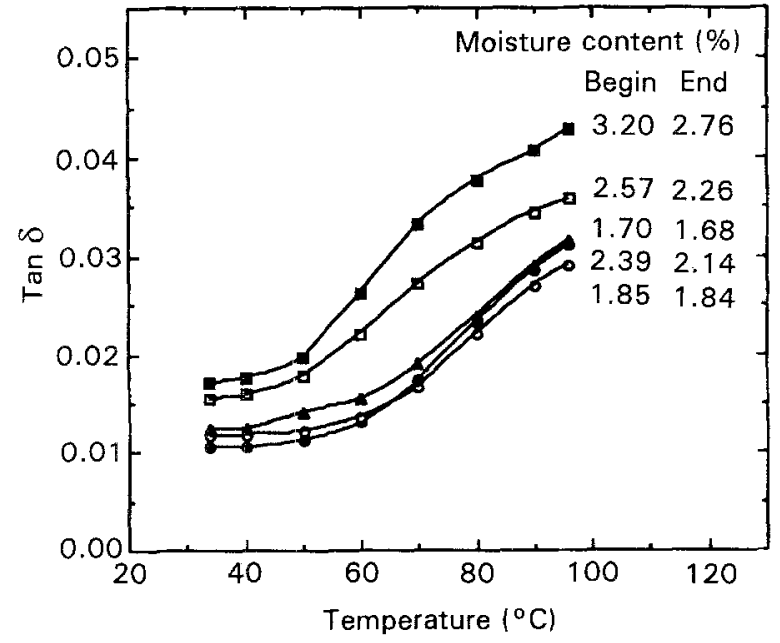

Figure 4 Loss tangents, $\tan \delta$, of MDF cement versus temperature at an oscillation frequency of $1 \mathrm{~Hz}$ for various moisture contents. (The moisture contents at the beginning and end of the scan from low to high temperature are indicated.)

$34-96^{\circ} \mathrm{C}$, and no peaks or shoulders were observed near or below $64^{\circ} \mathrm{C}$, which is the glass transition temperature of dry PVA. The loss tangent tended to shift to lower temperatures as moisture content increased, though the data were not monotonic. The $\tan \delta$ curves for the three specimens with least moisture were observed to cross one another as the temperature approached the boiling point of water $\left(100^{\circ} \mathrm{C}\right)$.

\section{Discussion}

\subsection{Viscoelasticity}

With increasing moisture content, the storage modulus $G^{\prime}$ of MDF cement was found to decrease in both the frequency (Fig. 1) and temperature scans (Fig. 3). The loss tangent $(\tan \delta)$ also tended toward higher values as the moisture content increased. These indicate that moisture enhances the viscous response of the material. This can be mainly attributed to the PVA binder phase, which softens in the presence of moisture. MDF also softens and becomes more viscous at lower oscillation frequencies or higher temperatures like other viscoelastic materials $[12,13]$. The conventional viscoelasticity parameters of time and temperature are also applicable to MDF.

Tan $\delta$ normally reaches local maxima at transition temperatures. No maximum was found within the temperature range scanned $\left(34-96^{\circ} \mathrm{C}\right)($ see Fig. 4). The cross-linking believed to be present in MDF may shift the polymer glass transition temperature, $T_{\mathrm{g}}$, to higher values, however. Moisture, in contrast, reduces the transition temperature. The lack of transition temperature in the vicinity of the regular $T_{\mathrm{g}}$ of PVA, $64^{\circ} \mathrm{C}$, indicates that the PVA in MDF has been altered by the presence of the cement and no longer behaves as it does in the free state.

As seen in Fig. 1, the behaviour of low moisture content MDF at low frequency is similar to that with higher moisture content at higher frequency. Moisture and frequency affect the stiffness in a similar manner, suggesting a superposition of the curves affected by these two factors. The curves in Fig. 1, obtained at

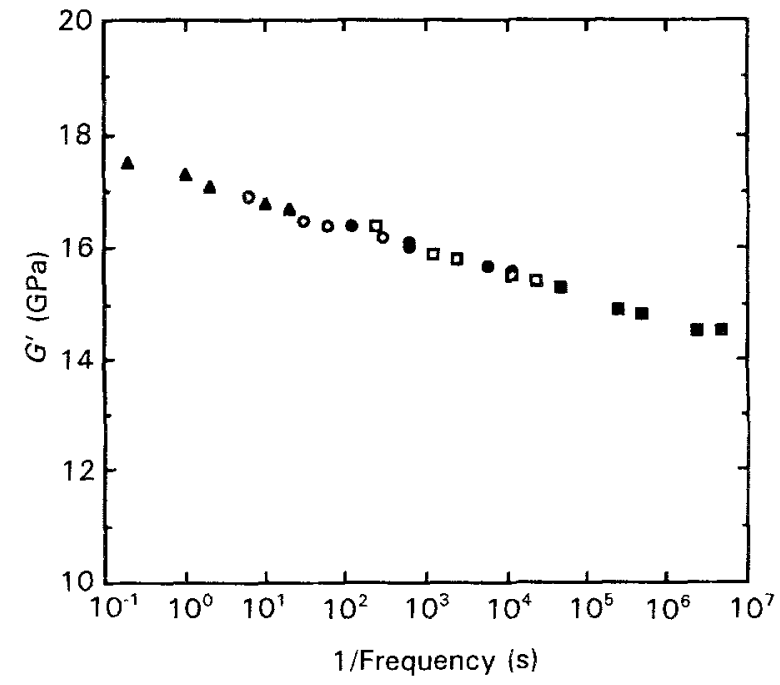

Figure 5 Superposition of curves from Fig. 1 with the curve for lowest moisture content $(1.70 \%)$ used as the reference. (A) $1.70 \%$, (O) $1.85 \%$, (O) $2.39 \%$, (口) $2.57 \%$, (ㄷ) $3.20 \%$.

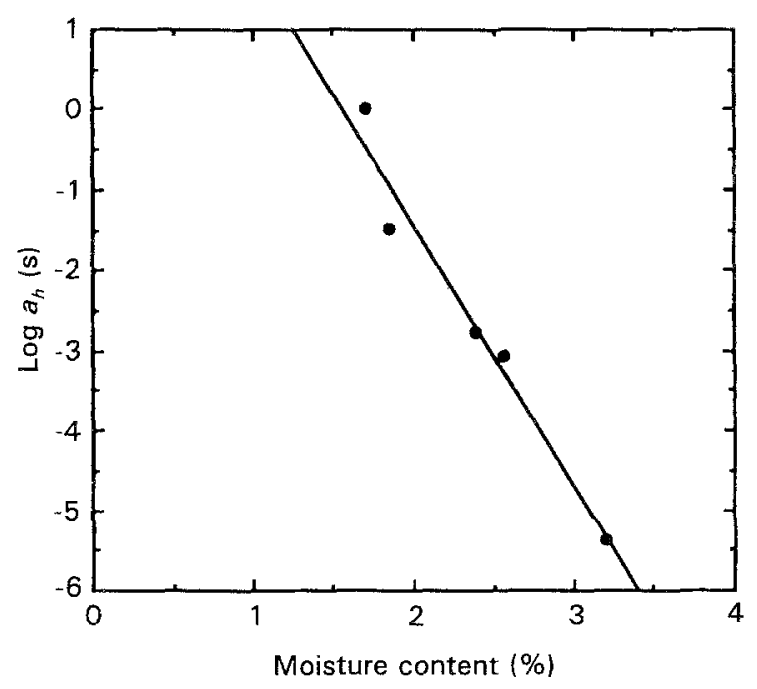

Figure 6 The shift factors versus moisture content for the superposition shown in Fig. 5.

$34^{\circ} \mathrm{C}$, have been shifted to superimpose them on that for the lowest moisture content $(1.70 \%)$. These form a single curve (see Fig. 5). The shift factors, $\log a_{h}$, of this time-moisture superposition versus moisture at $34^{\circ} \mathrm{C}$ are shown in Fig. 6 . The data points range over five decades of time to accommodate the span of moisture in these experiments. For MDF in the moisture range of the present experiments, the shift factors seem to lie on a straight line given by

$$
\log a_{h}=b_{1}-b_{2} h
$$

where $h$ is the moisture content. With $a_{h}$ in seconds and $h$ in per cent, the parameters $b_{1}$ and $b_{2}$ from least squares fitting to the data in Fig. 6 have the values of 5.1 and 3.3 , respectively.

A similar superposition method can be applied to temperature and moisture. As seen in Fig. 3, increasing moisture and increasing temperature cause a similar effect on the viscoelastic behaviour of MDF. A given stiffness can be achieved in an MDF specimen at higher moisture content by reducing temperature. When the storage modulus versus temperature curves 


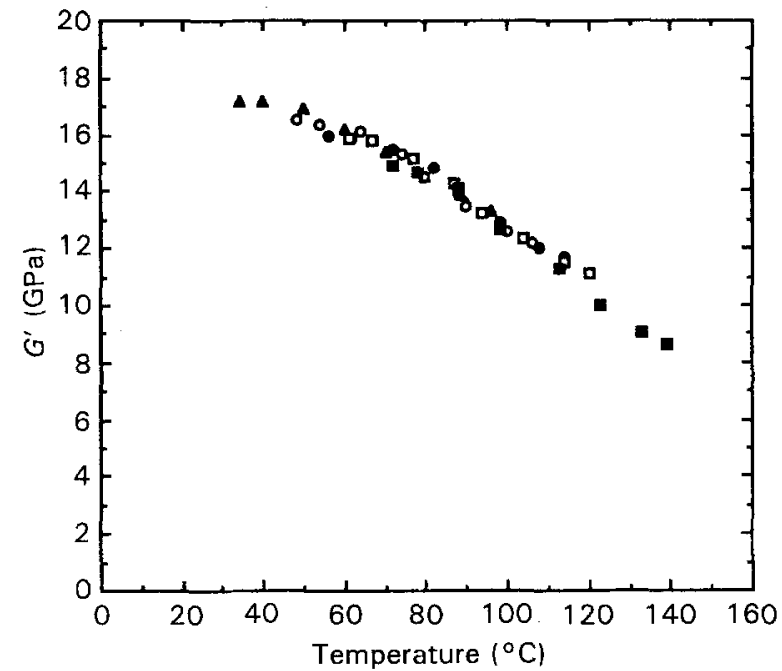

Figure 7 Superposition of curves from Fig. 3 with the curve for lowest moisture content $(1.70 \%)$ used as the reference. Moisture contents, begin/end: (A) $1.70 \% / 1.68 \%$, (O) $1.85 \% / 1.84 \%$, ( $2.39 \% / 2.14 \%$, (口) $2.57 \% / 2.26 \%$, (西) $3.20 \% / 2.76 \%$.

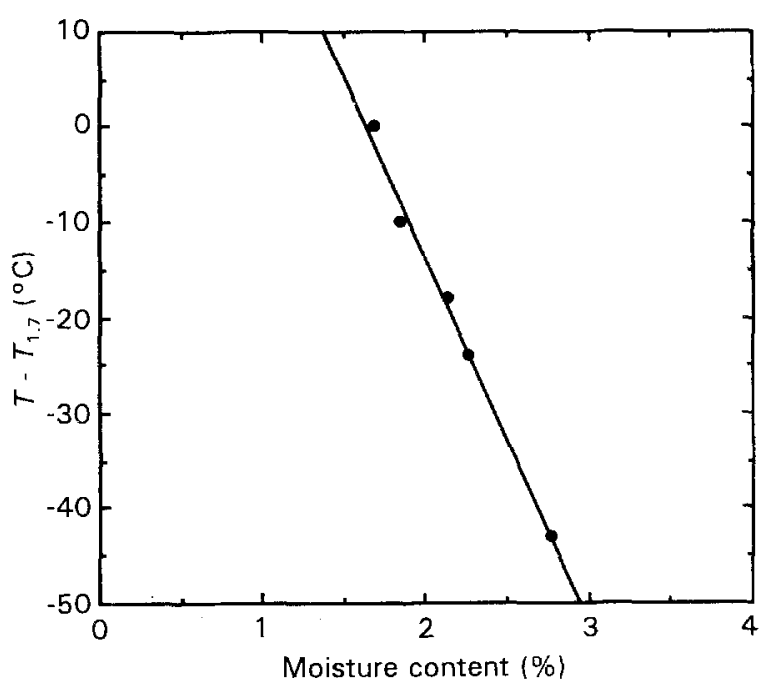

Figure 8 The shift factors versus moisture content for the superposition shown in Fig. 7.

at $1 \mathrm{~Hz}$ for different moisture contents are shifted horizontally to the lowest moisture content curve $(1.70 \%)$, they form a reasonably good master curve as seen in Fig. 7. With the lowest moisture content of $1.70 \%$ as reference, the temperature shift factors $\left(T-T_{1.7}\right)$ for this temperature-moisture superposition versus moisture are shown in Fig. 8. The temperature shifts by more than $40^{\circ} \mathrm{C}$ over the range of moisture contents in these experiments. As with the shift factors for the time-moisture superposition, the shift factors for the temperature-moisture superposition seem to be a linear function of moisture content in the range of these experiments

$$
T-T_{1.7}=d_{1}-d_{2} h
$$

where $h$ is again the moisture content. With $h$ in per cent, the parameters $d_{1}$ and $d_{2}$ from least squares fitting to the data in Fig. 8 have the values of 65.8 and $39.2^{\circ} \mathrm{C}$, respectively.

To effect the superposition with respect to temperature, the data obtained at $60^{\circ} \mathrm{C}$ and below were assumed to have the "begin" moisture content, and the data obtained above $60^{\circ} \mathrm{C}$ were taken to have the "end" moisture content. The ability to effect superposition did not depend on this particular scheme, however. The use of an average moisture content gave an equally satisfactory superposition.

The plasticizing effect of small molecules on polymers has received much attention $[13,14]$. The incorporation of a plasticizer into a polymer lowers the glass transition temperature of the polymer. The depression of the glass transition temperature has been found to be generally describable by the linear relation with respect to the diluent content [13]

$$
T_{\mathrm{g}}=T_{\mathrm{g} 1}-k w_{2}
$$

where $T_{\mathrm{g}}$ and $T_{\mathrm{g} 1}$ are the glass transition temperatures of the plasticized and unplasticized polymers, respectively, $w_{2}$ is the weight fraction of the diluent, and $k$ is a coefficient that depends on both the plasticizer and polymer. In the range of moisture contents studied $(1.70 \%-3.20 \%), w_{2}$ is approximately equal to the moisture content $h$. By choosing the moisture content of $1.70 \%$ as reference and rearranging Equation 3, it can be put in the same form as Equation 2. Thus, the time-temperature-moisture superposition found for MDF cement is consistent with the general behaviour of plasticized polymers.

\subsection{Mechanical models}

An increase in temperature to above $100^{\circ} \mathrm{C}$ was found to cause the shear modulus of MDF cement in the presence of moisture, as measured by dynamic mechanical analysis (DMA), to decrease to about one-half of the room-temperature value (Fig. 3). In contrast, the shear modulus of the polymer binder in MDF (PVA) decreases over a similar change in temperature to about $1 / 30$ th of the room-temperature value (Fig. 9). This large difference can be attributed to the microstructure or arrangement of the organic and inorganic components in MDF composite. This suggests that a structure of the composite can be deduced

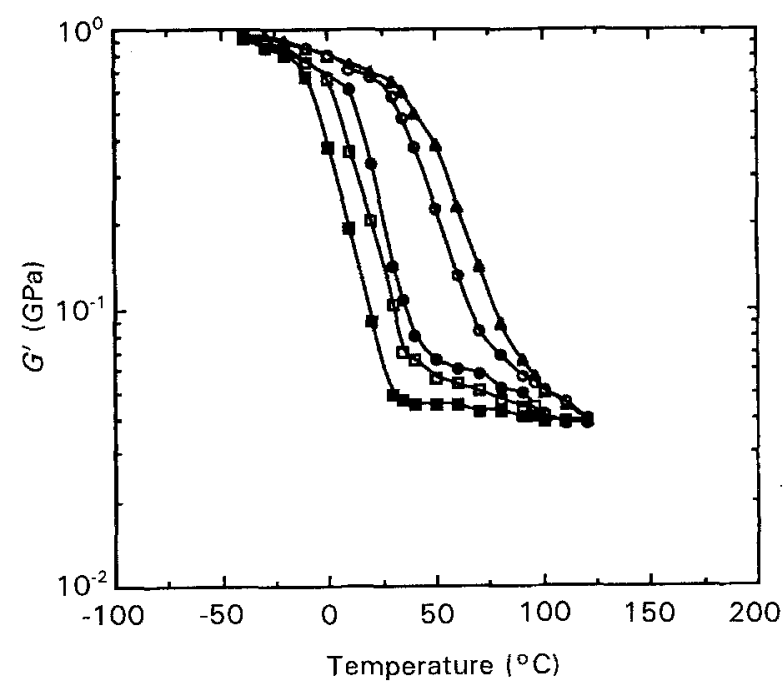

Figure 9 Normalized shear storage moduli, $G^{\prime}$, of PVA as a function of temperature for various moisture contents: $(A) 0 \%,(O)$ $0.96 \%$, (○) $5.01 \%$, (口) $9.6 \%$, (口) $15.16 \%$. 
from a comparison of mechanical models for the cement with the observed viscoelastic response. MDF is a multi-component, multi-phase composite material that consists of PVA and calcium aluminates (CA), each with very different moduli. The following values were used for the following mechanical modelling. The shear modulus of PVA was assumed to range between 0.035 and $1 \mathrm{GPa}$ as moisture and temperature are changed, the same as is indicated in Fig. 9. Because a measured shear modulus for CA could not be found in the literature, the shear modulus was estimated to be similar to that of soda-lime glass, namely $30 \mathrm{GPa}$, and this was assumed to remain constant over the range of temperatures and moisture contents used. (Both the results of this modelling and theoretical considerations support the choice of this modulus.) The dry, low-temperature moduli ratio of CA/PVA is therefore about 30. For MDF consisting of 7 parts by weight PVA and 100 parts of CA, assuming the specific gravities of 1.26 and 3.2, respectively, the volume fractions are calculated to be $V=0.85 \mathrm{CA}$ and $(1-V)=0.15$ PVA. For simplicity, the property values of the hydrates were assumed to be nearly the same as the anhydrous CA. The volume change due to moisture absorption was neglected.

Mechanical models have often been applied to composites and polymer blends [15-18]. Several possible models are shown in Fig. 10. The orientation of the diagrams with respect to the stress and strain is indicated by the arrows in Fig. 10a. Shear stress and strain are assumed to occur in the plane of the diagram, in a vertical direction.

The two simplest models have the inorganic and polymer phases assembled either in series (Fig. 10b),

\section{Number of}

elements

MDF

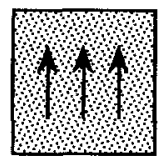

(a)
2

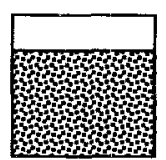

(b)

3

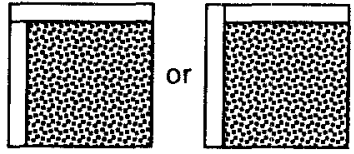

(d)

4
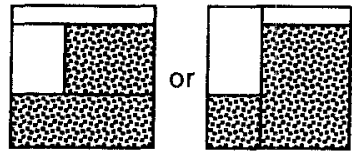

(f)

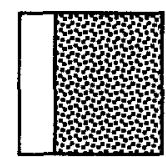

(c)

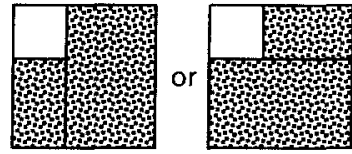

(e)

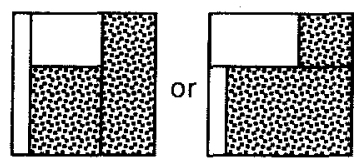

(g) $\square=$ Organic phase

Figure 10 Mechanical models for MDF. for which the shear modulus is

$$
\frac{1}{G}=\frac{V}{G_{\mathrm{c}}}+\frac{(1-V)}{G_{\mathrm{p}}}
$$

or in parallel (Fig. 10c), for which the shear modulus is

$$
G=V G_{\mathrm{c}}+(1-V) G_{\mathrm{p}}
$$

$V$ is the volume fraction of the cement phase; $G, G_{c}$ and $G_{\mathrm{p}}$ are the moduli of the MDF composite, the cement phase, and the polymer phase, respectively. The series model corresponds to the polymer surrounding each inorganic particle and bonding them together. The parallel model corresponds to the polymer and inorganic both being continuous, or co-continuous, phases. Comparisons of the storage modulus obtained experimentally from the dynamic mechanical analysis versus temperature, with that predicted by the simple parallel and series models, are shown in Fig. 11. Neither model fits the data at all well.

Another model that has been successfully applied to predicting the properties of composites with discontinuous fillers dispersed in a continuous matrix is the Halpin-Tsai equation [19]. The shear modulus given by this model is

$$
\frac{G}{G_{\mathrm{p}}}=\frac{1+\eta V \zeta}{1-\eta V}
$$

where $\eta=\left(G_{\mathrm{c}} / G_{\mathrm{p}}-1\right) /\left(G_{\mathrm{c}} / G_{\mathrm{p}}+\zeta\right)$, and $\zeta=2$ for a filler aspect ratio of 1 . The Halpin-Tsai equation represents a model similar to that shown in Fig. 10d. As seen from the comparison of the predictions of the Halpin-Tsai equation with experimental data in Fig. 11, there is broad discrepancy, though micrographs of MDF [7] suggest a structure of inorganic particles bonded by a PVA matrix. The Halpin-Tsai equation may be successful for random fillers over a wide range of aspect ratios, but it is only good for a minor quantity of independent reinforcements dispersed in a continuous matrix.

A composite model where a minor volume fraction of organic phase is dispersed in a major fraction of inorganic phase is shown in Fig. 10e. (The PVA portion is unshaded and the calcium aluminates are

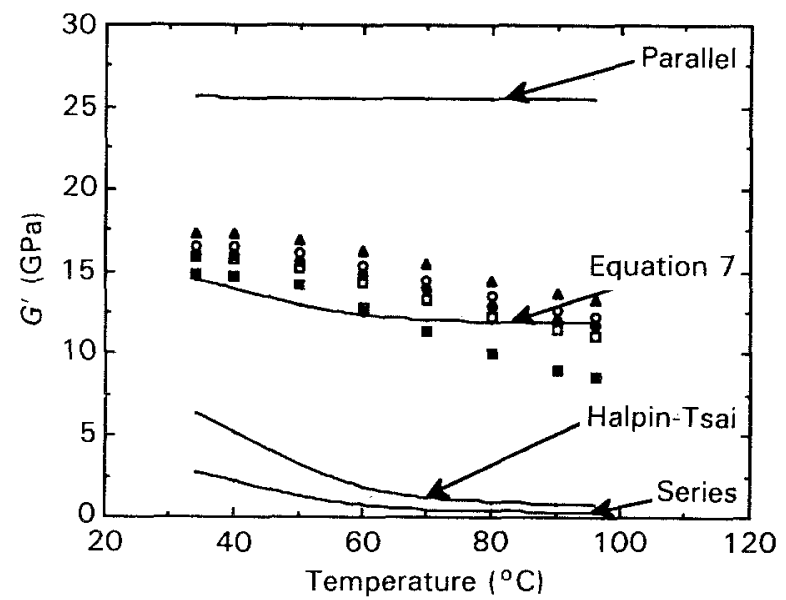

Figure 11 Comparison of the shear storage moduli predicted by mechanical models of up to three elements with the experimental results from Fig. 3. 


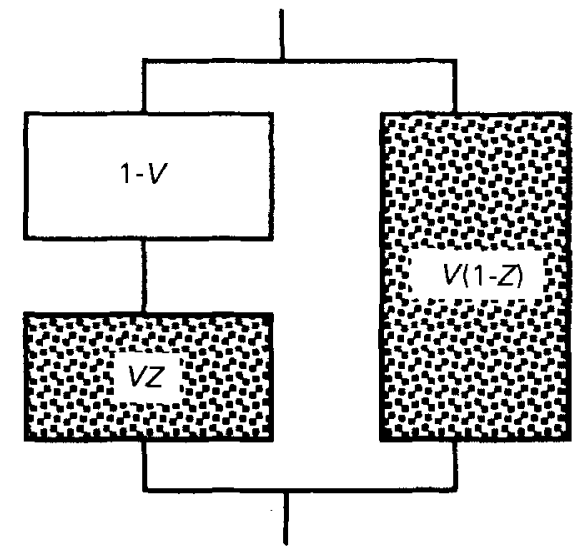

(a)

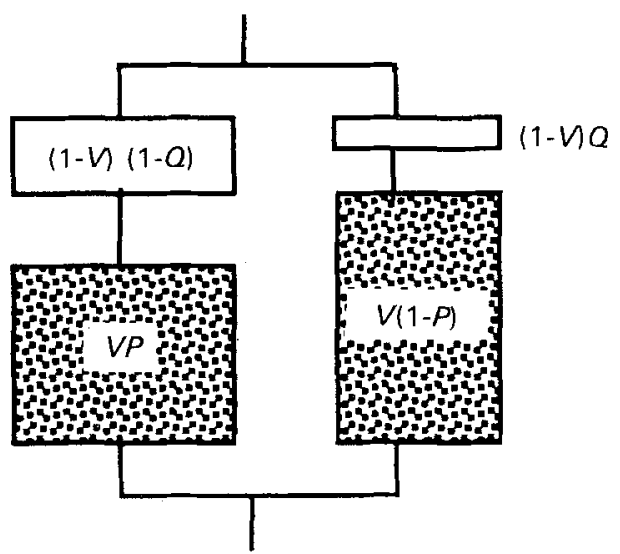

(b)

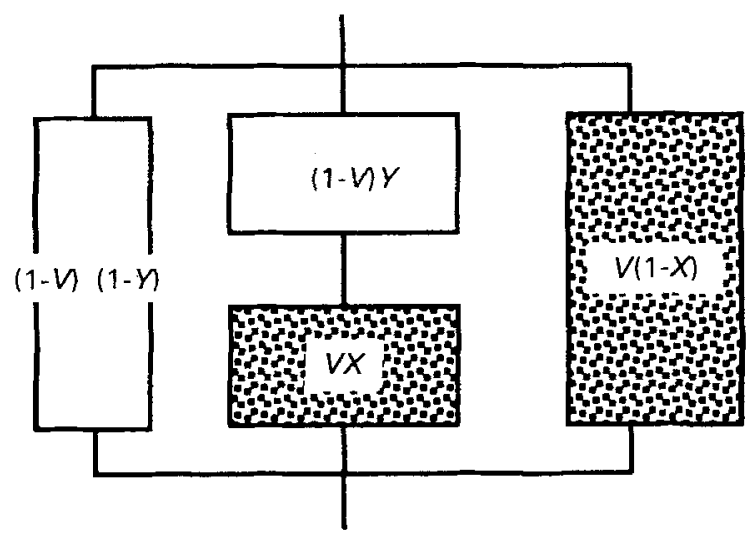

(c)

Figure 12 Parameter assignment for models shown in Fig. 10e-g, corresponding to (a-c), respectively.

shaded.) This arrangement is shown also in Fig. 12a, where the volume fractions of calcium aluminates and PVA in MDF are denoted by $V$ and $(1-V)$, respectively. The portion of the calcium aluminates in series with PVA are denoted by $Z$, and the rest of the calcium aluminates, $(1-Z)$, are parallel to it. The composite modulus derived from this physical model is

$$
\begin{aligned}
G= & V(1-Z) G_{\mathrm{c}} \\
& +(1-V-V Z)^{2} /\left(\frac{1-V}{G_{\mathrm{p}}}+\frac{V Z}{G_{\mathrm{c}}}\right)
\end{aligned}
$$

The storage moduli predicted fall within the range of the experimental results, as seen in Fig. 11. The model represents a structure where some of the calcium aluminates are directly attached, allowing some of the load to be borne through them. The rest of the calcium aluminates particles are in series with PVA. Although a part of the continuous PVA matrix may run from top to bottom, it is assumed to carry so little load that it is neglected in this model. The predictions of this model are superior to those of all other models of up to three elements, though the fitting of the data is not completely satisfactory. The connections or continuity through the inorganic phase probably arises from the hydration products.

Mechanical models containing four elements, as shown in Fig. $10 \mathrm{f}$ and g, may also be considered. For the mechanical model shown in Fig. 10f, shown also in Fig. $12 \mathrm{~b}$, a portion of the inorganic phase, $P$, is assumed to be in series with a major part of the PVA. The latter is denoted by $(1-Q)$. The rest of the inorganic phase $(1-P)$ is in series with the rest of the PVA $(Q)$. Similar models for cement pastes and concrete have been reviewed by Pratt [20]. The shear modulus is expected to be

$$
\begin{aligned}
G= & {[V(1-P)+(1-V) Q]^{2} } \\
& /\left[\frac{V(1-P)}{G_{\mathrm{c}}}+\frac{(1-V) Q}{G_{\mathrm{p}}}\right] \\
& +[V P+(1-V)(1-Q)]^{2} \\
& /\left[\frac{V}{G_{\mathrm{c}}}+\frac{(1-V)(1-Q)}{G_{\mathrm{p}}}\right]
\end{aligned}
$$

The parameters of this model were optimized by fitting the equation to the centre of the band of experimental data in Fig. 3, to material with $2.39 \%$ moisture, which is normal MDF, and the result is shown in Fig. 13. The optimized parameters for this moisture content are $P=0.33$ and $Q=0.0032$. The parameters

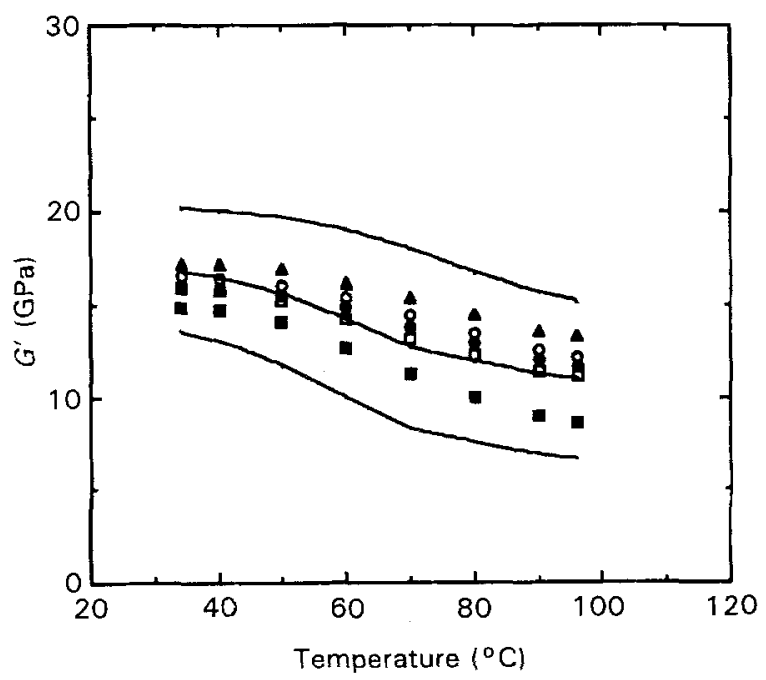

Figure 13 Comparison of shear storage moduli predicted by the mechanical model shown in Fig. $10 \mathrm{f}$ with the experimental results from Fig. 3. 
corresponding to $0 \%$ moisture are $P=0.2$ and $Q=0.003$, which give the upper curve in Fig. 13, and the parameters corresponding to moisture saturation $(\approx 4 \%$ ) are $P=0.45$ and $Q=0.005$, which give the lower curve.

The above model seems to be consistent with the microstructure of MDF as described by Popoola et al. [11], who found that MDF cement could be described as consisting of at least three phases: unhydrated calcium aluminates (CA), poly(vinyl alcohol) (PVA), and an interphase consisting of calcium aluminate hydrates and PVA. The above model represents the arrangement of these phases. The main load path is through the inorganic phases $(1-P)$ that includes the hydrates, and these are in series with thin layers of PVA $(Q)$, as shown on the right arm of Fig. 12b. An auxiliary load path is through the major part of the PVA $(1-Q)$ that is in series with unhydrated calcium aluminates $(P)$, the left arm of Fig. $12 \mathrm{~b}$. The hydrates are assumed to be involved in the right arm of Fig. $12 b$ only because that is where their effect is critical.

The implications of the above model can be compared with other data. An estimate from the model of the fraction of the inorganic phase that has been converted to hydrates can be made as follows: the volume fractions of the inorganic phases (assumed to be unhydrated calcium aluminates) and PVA in the left arm of Fig. $12 \mathrm{~b}$ are $V P=(0.85 \times 0.33)=28 \%$ and $(1-V)(1-Q)=(0.15 \times 0.9968)=15 \%$, respectively. This means that the unhydrated calcium aluminates occupy about $28 \% /(28 \%+15 \%)=0.65$ of the volume of the left arm. Because this fraction is expected to be maintained in the right arm, the combined volume fractions of hydrates and PVA in the right arm are $(1-0.65)(1-0.28-0.15)=20 \%$. The volume fraction of PVA in this arm, $(1-V) Q=0.15 \times$ $0.0032=0.05 \%$, is negligible, however, so that the fraction of hydrates relative to the total inorganic phases is $20 \% / 85 \%=23.5 \%$. This value is similar to about $21 \%$ that was computed and reported recently by Lewis and Kriven [21].

Another test is the ratio between PVA and unhydrated calcium aluminates in series without the embedded hydrates, in the left arm of Fig. $12 \mathrm{~b}$. The ratio is estimated to be $15 \% / 28 \% \approx 0.5$. This implies that the thickness of the PVA layer is about one-half that of the calcium aluminate particles. But micrographs of sections through the MDF suggest that this is too large. Another negative feature of this model is that the PVA is assumed to be discontinuous. This is in conflict with the percolative nature of the binder.

A model based on a connected network for the inorganic phase is shown in Figs $10 \mathrm{~g}$ and 12c. The inorganic and the organic phases are combined in both parallel and series. In this way, there are two continuous phases coexisting in the system, partially in parallel and partially in series with each other. Parameters $X$ and $Y$ are assigned to the series portions of the inorganic and the organic phases, respectively (Fig. 12c). The rest of the inorganic and organic phases, $(1-X)$ and $(1-Y)$, are the parallel portions. The composite modulus is

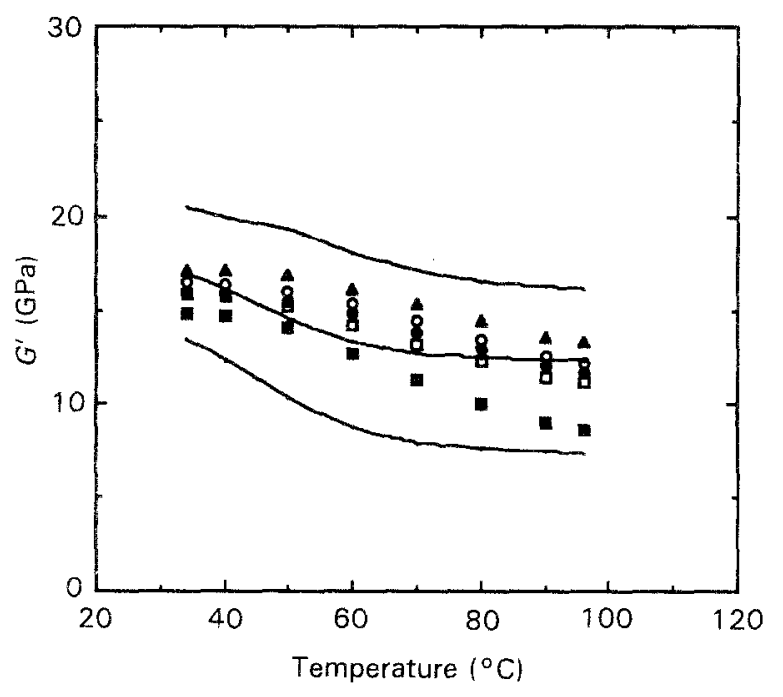

Figure 14 Comparison of shear storage moduli predicted by the mechanical model shown in Fig. $10 \mathrm{~g}$ with the experimental results from Fig. 3.

$$
\begin{aligned}
G= & (1-V)(1-Y) G_{\mathrm{p}}+V(1-X) G_{\mathrm{c}} \\
& +[(1-V) Y+V X]^{2} \\
& /\left[\frac{(1-V) Y}{G_{\mathrm{p}}}+\frac{V X}{G_{\mathrm{c}}}\right]
\end{aligned}
$$

The parameters of this model were optimized by again fitting to the centre of the band of the experimental data in Fig. 3, to $2.39 \%$ moisture, which is normal MDF, and the result is shown in Fig. 14. The optimized parameters for this moisture content are $X=0.55$ and $Y=0.06$.

The parameters corresponding to $0 \%$ moisture are $X=0.4$ and $Y=0.05$, which give the upper curve in Fig. 14, and the parameters corresponding to moisture saturation $(\approx 4 \%)$ are $X=0.75$ and $Y=0.12$, which give the lower curve.

The parameters $X=0.55$ and $Y=0.06$ lead to the conclusion that $45 \%(=1-X)$ of the inorganic phase is continuous. This is consistent with the fact that MDF retains some strength after the PVA is burned away at $500^{\circ} \mathrm{C}$ [3]. This temperature would not have been high enough to induce sintering for the inorganic phase. The strength retention suggests a connected skeleton for the inorganic phase. However, the degree of continuity estimated from the burn-out experiment, about $10 \%[3,21]$, is much lower than was estimated above, which is about $45 \%$. This difference could arise from artefacts like shrinkage cracks and the dehydration of the hydrates being introduced during burn-out. Hence, $45 \%$ may be a better estimate for the degree of continuity, than that is given by the retained strength after burn-out.

\section{Conclusion}

The viscoelastic behaviour of MDF cannot be predicted by either simple, two-element parallel or series models or the Halpin-Tsai equation. The threeelement model in which the inorganic phase is partially continuous through the material but the PVA is not, shown in Fig. 10e, is able to give a much improved fit to the data, though the fit is not completely 
satisfactory. Among four-element models, both the model in which neither the inorganic phase nor the PVA are continuous through the material, shown in Fig. 10f, and the model in which the inorganic and PVA phases are both partially continuous through the material, shown in Fig. $10 \mathrm{~g}$, predict adequate viscoelastic responses within experimental error for MDF (Figs 13 and 14). But for the model in which neither the inorganic phase nor the PVA are continuous through the material (Fig. 10f), the proportions of the phases required to obtain an adequate fit with the viscoelastic data are not in complete agreement with those determined by other means. This model also is not consistent with the strength retention found after polymer burn-out. However, the four-element model in which the inorganic and PVA phases are both partially continuous through the material (Fig. $10 \mathrm{~g}$ ) is consistent with known experimental data. When applied to the viscoelastic behaviour found for MDF, this model suggests that roughly $45 \%$ of the inorganic phases are bonded together directly, without PVA.

\section{Acknowledgement}

We thank Professor Sherman D. Brown and Mr Steve K. Holzgraefe, University of Illinois, Urbana-Champaign, for their help in preparing the MDF specimens, and Professor Jennifer A. Lewis for useful discussions. This study was supported by the NSF Center for Science and Technology for Advanced Cement-Based Materials (ACBM), NSF Grant DMR-8808432.

\section{References}

1. J. D. BIRCHALL, A. J, HOWARD and K. KENDALL, Nature 289 (1981) 388.

2. W. SINCLAIR and G. W. GROVES, J. Mater. Sci. 20 (1985) 2846.
3. C. S. POON, L. E. WASSELL and G. W. GROVES, Mater. Sci. Technol. 4 (1988) 993.

4. C. M. CANNON and G. W. GROVES, J. Mater. Sci. 21 (1986) 4009.

5. N. B. EDEN and J. E. BAILEY, ibid. 19 (1984) 2677.

6. K. TOYoshima, in "Polyvinyl Alcohol", edited by C. A. Finch (Wiley, London, 1973) 339.

7. J. D. BI RCHALL, Phil. Trans. R. Soc. Lond. A301 (1983) 31

8. S. A. RODGER, A. S. BROOKS, W. SINCLAIR, G. W. GROVES and D. D. DOUBLE, J. Mater. Sci. 20 (1985) 2853.

9. D. M. ROY, Science 235 (1987) 651

10. R. N. EDMONDS and A. J. MAJUMDAR, J. Mater. Sci. 24 (1989) 3813.

11. O. O. POPOOLA, W. M. KRIVEN and J. F. YOUNG, J. Am. Ceram. Soc. 74 (1991) 1928.

12. N. G. McCRUM, B. E. READ and G. WILLIAMS, "Anelastic and Dielectric Effects in Polymeric Solids" edited by R. F. Gould (Wiley, London, 1967).

13. J. D. FER R Y "Viscoelastic Properties of Polymers", 3rd Edn. (Wiley, New York, 1980).

14. M. C. SHEN and A. V. TOBOLSKY, in "Plasticization and Plasticizer Processes", Advances in Chemistry Series 48 (American Chemical Society, Washington, D.C., 1965) p. 27.

15. L. E. NiElsen, "Mechanical Properties of Polymers and Composites", Vol. 2 (Marcel Dekker, New York, 1974).

16. J. A. MANSON and L. H. SPERLING, "Polymer Blends and Composites" (Plenum Press, New York, 1976).

17. R. A. DICKIE, in "Polymer Engineering Composites", edited by M. O. W. Richardson (Applied Science, London, 1977) p. 155.

18. L. E. NIELSEN, "Predicting the Properties of Mixtures Mixture Rules in Science and Engineering" (Marcel Dekker, New York, 1978).

19. K. K. CHAWLA, "Composite Materials" (Springer, New York, 1987).

20. P. L. PRATT, in Microstructural Development During Hydration of Cement, "Materials Research Society Symposium Proceedings, Vol. 85" edited by L. J. Struble and P. W. Brown (The Material Research Society, Pittsburgh, PA, 1987) p. 145.

21. J. A. LEWIS and W. M. KRIVEN, MRS Bull. 18(3) (1993) 72.

Received 26 January

and accepted 1 September 1993 